\title{
Pyruvate dehydrogenase kinase 1 and carbonic anhydrase IX targeting in hypoxic tumors
}

\author{
M. KERY*, N. ORAVCOVA*, S. RADENKOVIC, F. IULIANO, J. TOMASKOVA, T. GOLIAS* \\ Institute of Virology, Biomedical Research Center, Slovak Academy of Sciences, Bratislava, Slovak Republic \\ ${ }^{*}$ Correspondence: viruteho@savba.sk

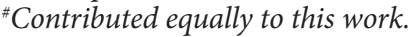

Received May 31, 2018 / Accepted June 12, 2018

\begin{abstract}
Pyruvate dehydrogenase kinase 1 (PDHK1) and carbonic anhydrase IX (CAIX) are some of the most hypoxia-inducible proteins associated with tumors, implicated in glucose metabolism and $\mathrm{pH}$ regulation, respectively. They both appear to be necessary for model tumor growth, and their high level of expression in human tumors predicts poor patient outcome. Another thing they have in common is that hypoxia not only induces their expression but also their enzymatic activity. This work therefore simultaneously targets these two hypoxia-inducible proteins either pharmacologically or genetically in vitro and in vivo, leading to decreased cancer cell survival and significantly slower model tumor growth. It also suggests that CAIX and PDHK1 are important for cells originating from a colorectal primary tumor, as well as from its metastasis. Moreover, our analysis reveals a unique relationship between these two HIF-1 target genes. In conclusion, the attributes of PDHK1 and CAIX predict them to be promising targets for the design of new, specific inhibitors that could negatively influence tumor cell proliferation and survival, or increase efficacy of standard treatment regimens, and at the same time avoid normal tissue toxicity.
\end{abstract}

Key words: tumor microenvironment, hypoxia, pyruvate dehydrogenase kinase 1, carbonic anhydrase IX

Hypoxia, or decreased tissue oxygen partial pressure, is a well-known phenomenon in cancer. Its primary cause is worsened blood supply to tumors rapidly outgrowing the adjacent vasculature but it can also be caused by fluctuating blood flow in the aberrant newly formed tumor vessels [1]. Cancer cells in the hypoxic environment make a coordinated response to the oxygen-restricted conditions. The HIF (hypoxia-inducible factor) transcription factor, a heterodimer consisting of an oxygen-dependent $a$ subunit and a constitutive $\beta$ subunit, is the main mediator of this response. The molecular mechanism underlying the selectivity for hypoxia is the oxygen-dependent degradation domain (ODDD) of HIF- $\alpha$, which is responsible for its normoxic degradation and hypoxic stabilization [2]. Under normoxic conditions, HIF-a protein is rapidly targeted for ubiquitin-mediated degradation by the proteasome, because the HIF-a ODDD possesses two proline residues which are hydroxylated in the presence of oxygen by prolyl hydroxylases. The hydroxyproline residues are then recognized by von Hippel-Lindau tumor suppressor protein ( $\mathrm{pVHL}$ ), the recognition element of a multicomponent ubiquitin ligase that ubiquitinates and targets HIF- $\alpha$ for proteolysis by the ubiquitin-proteasome pathway [3]. During hypoxia, when oxygen as a substrate for prolyl hydroxylases becomes limited, HIF-a protein evades hydroxylation and degradation, and is stabilized. Then, it translocates to the nucleus along with HIF- $\beta$ where they generate a transcriptional response to hypoxia together by binding to hypoxia-response elements (HREs) in the promoter/enhancer regions of target genes [3]. About 100 direct HIF target genes have been identified so far. For example, HIF-1 isoform activation shifts metabolism from oxidative to glycolytic in both hypoxic as well as oxygenated regions of the tumor (Warburg effect) by upregulating expression of glycolytic enzymes and glucose transporters [4]. Considering the relative inefficiency of glycolysis in ATP production compared to mitochondrial oxidative phosphorylation (OXPHOS), this adaptation in cellular bioenergetics does not appear logical. But it seems that by switching to glycolytic metabolism, cancer cells bring the demand for oxygen closer to the restricted supply and conserve it for cells even further away from its source [5], as well as provide intermediates for anabolic reactions necessary for sustained proliferation [6], while maintaining sufficient energy production and benefiting the tumor as a whole. 
Apart from directly upregulating glycolysis, mitochondrial function is also actively downregulated by HIF-1, through the induction of pyruvate dehydrogenase kinase 1 and 3 (PDHK1, PDHK3) [7-9] that inhibit pyruvate dehydrogenase (PDH), a gate-keeping enzyme to the tricarboxylic acid-cycle (TCA) in mitochondria. PDH is inhibited by phosphorylation of its three inhibitory serine residues (Site $1=$ Ser293, Site $2=$ Ser300, Site 3 = Ser232). Interestingly, PDHK1 isoform is indispensable for the phosphorylation of serine 232 [10]. When pyruvate derived from glucose cannot be converted to acetyl-CoA by PDH, it is preferentially broken down to lactate by lactate dehydrogenase in the cytoplasm (LDH; another HIF-1 target gene [11]). Therefore, PDHKs likewise support glycolysis at the expense of OXPHOS. However, excess lactate poses a threat to the neutral intracellular environment. Apart from lactic acid, other significant sources of acidity in tumors are protons and carbon dioxide [12]. HIF induces a number of proton transporters [13], and $\mathrm{CO}_{2}$ is neutralized in a reaction generating $\mathrm{HCO}_{3}{ }^{-}$and $\mathrm{H}^{+}$by carbonic anhydrase enzymes (CAs). Of these, transmembrane CAIX (with an extracellular catalytic site) is almost exclusively related to cancer and is also a HIF-1 target gene [14]. Intracellular $\mathrm{CO}_{2}$ freely diffuses to extracellular space where CAIX converts it to bicarbonate and proton, thus contributing to acidification of extracellular $\mathrm{pH}$. But at the same time, it prevents acidification of the intracellular milieu, because the CAIX-generated bicarbonate is transported into the cell by bicarbonate transporters, where it neutralizes intracellular $\mathrm{pH}$ [15]. Standard neutral intracellular $\mathrm{pH}$ combined with low extracellular $\mathrm{pH}$ promote an aggressive tumor phenotype.

Hypoxia-inducible PDHK1 and CAIX both appear to be necessary for model tumor growth, and their high level of expression in human tumors predicts poor patient outcome [16-18]. Another characteristic they share is that hypoxia not only induces their expression but also their activity $[10,19-21]$. This work therefore attempts to target these two highly HIF-1 responsive genes in vitro as well as in vivo, because their attributes predict them to be promising new targets for the design of specific inhibitors that could negatively influence tumor cell proliferation and survival, and at the same time avoid normal tissue toxicity or increase efficacy of standard treatment regimens.

\section{Materials and methods}

Cell lines and cell culture. Human colorectal carcinoma RKO cell line, RKO control, RKO shPDHK1, and human pancreatic carcinoma SU.86.86 control and SU.86.86 shPDHK1 cell lines were a kind gift from Dr. Nicholas Denko (Ohio State University, Columbus, OH). Human colorectal adenocarcinoma SW480 cell line and metastatic SW620 cell line derived from the same patient were purchased from ATCC. Cells were grown in DMEM medium (Merck) with $10 \%$ FCS in high glucose $(25 \mathrm{mM})$ or low glucose $(5 \mathrm{mM})$.
Hypoxia $\left(0.5-1 \% \mathrm{O}_{2}\right)$ was achieved in an Invivo 2 humidified hypoxia workstation (Ruskinn Technologies).

Generation of knockout cell lines. RKO knockout cell lines were generated using the CRISPR/Cas9 technique. To create PDHK1 knockouts, RKO cells were co-transfected with pX335-crisprPDHK1 A, pX335-crisprPDHK1 B, and pTK-Hygro using TurboFect (Thermo Fisher Scientific), followed by selection in $500 \mu \mathrm{g} / \mathrm{ml}$ hygromycin B (SigmaAldrich) (control cells were co-transfected with empty pX335 vector and pTK-Hygro). To create CAIX knockouts and double PDHK1-CAIX knockouts, RKO cells and RKO PDHK1 knockout cells, respectively, were transfected with pX459-crisprCAIX using TurboFect, followed by selection in $1 \mu \mathrm{g} / \mathrm{ml}$ puromycin (Sigma-Aldrich) (control cells were transfected with empty pX459 vector). Drug-resistant colonies were tested by Western blotting, and four positive clones were randomly pooled for study. The PDHK1 gRNA/ Cas9nickase pX335 plasmid generation was described previously [10]. The CAIX gRNA/Cas9nuclease plasmid (pX459-crisprCAIX) was generated by introducing targeting sequence 5' ATGCAGGAGGATTCCCCCTT 3' into the BbsI site in pSpCas9(BB)-2A-Puro (PX459) (Addgene plasmid \# 48139).

Cell viability assays. RKO cells were plated in a 96-well plate at 25000 cells/well and preincubated in hypoxia $\left(1 \% \mathrm{O}_{2}\right)$ overnight (to induce CAIX and PDHK1). The following day, inhibitors were added in fresh complete DMEM medium and incubated for 24 hours in normoxia. $100 \mu \mathrm{M} 4$-aminomethylbenzenesulfonamide (homosulfanilamide, HSFA) (SigmaAldrich) was used as a cell-impermeable carbonic anhydrase inhibitor and $10 \mathrm{mM}$ dichloroacetate (DCA) (SigmaAldrich) as a general pyruvate dehydrogenase inhibitor, plus their combination. To assess viability, CellTiter-Blue reagent (Promega) was added, cells were incubated for additional 3 hours, and fluorescence $\left(530(25)_{\mathrm{Ex}} / 590(35)_{\mathrm{Em}}\right)$ intensity was measured using the Synergy H4 microplate reader (BioTek).

For colony formation assay, 100 cells/well were plated in a 6-well plate in complete DMEM medium. The following day, medium was changed to high-glucose $(25 \mathrm{mM})$, highglutamine $(4 \mathrm{mM})$ DMEM medium with or without $1 \mathrm{mM}$ pyruvate, and low-glucose $(5 \mathrm{mM})$, low-glutamine $(1 \mathrm{mM})$ DMEM medium with or without pyruvate, and cells were left to grow for two weeks in normoxic conditions, after which the surviving colonies were fixed and stained with $0.25 \%$ crystal violet in ethanol (Sigma-Aldrich).

Western blotting and antibodies. Lysates were generated in RIPA buffer containing protease and phosphatase inhibitors $(5 \mathrm{mM}$ sodium fluoride, $2 \mathrm{mM} \beta$-glycerophosphate, $1 \mathrm{mM}$ sodium orthovnadate, $1 \mathrm{mM}$ phenylmethylsulfonyl fluoride, and Complete Mini protease inhibitor cocktail (Roche)), and $50 \mu \mathrm{g}$ of total proteins were separated in 8-10\% SDS-PAGE and blotted to PVDF. Antibodies used were for CAIX (M75 hybridoma medium produced in our laboratory, 1:5), PDHK1 (1:4000, Assay Designs), pSer232PDH (1:2000, Merck), total PDH (1:2000, Abcam), HIF-1a 
(1:500, BD), LDHA (1:10000, Cell Signaling Technology), $\beta$-actin (1:10000, Cell Signaling Technology), and $\alpha$-tubulin (1:10000, Sigma-Aldrich). Primary antibodies were detected with fluorochrome-labeled secondary antibodies (Li-Cor) and visualized on Li-Cor Odyssey or with HRP secondary antibodies (1:4000, Santa Cruz) and visualized with ECL on film.

Tumor growth delay. $2 \times 10^{6}, 3 \times 10^{6}$, or $4 \times 10^{6}$ cells were injected s.c. into the flanks of 6-16-week old female nude mice (six tumors per cell type, in triplicate experiments), and caliper measurements of two perpendicular diameters were used to monitor tumor volumes (volume $\left.=\left(\mathrm{d}_{1}\right) \times\left(\mathrm{d}_{2}\right)^{2} \times 0.52\right)$. Three random tumors per cell type were harvested in RIPA and analyzed by Western blotting as described above. Error bars represent the standard error of the mean. All animal experiments were performed following protocols approved by the State Veterinary and Food Administration of the Slovak Republic (project 292/16-221 g).

Statistical analyses. The data are presented as mean \pm standard deviation (SD) or standard error of the mean (SEM) where indicated. For comparison between two groups, they were tested by two-tailed Student's t-test and by two-way ANOVA for tumor growth curves. The p-value $<0.05$ was considered significant. The analyses were performed using Prism, version 7 (GraphPad, La Jolla, CA).

\section{Results}

PDHK1 and CAIX expression pattern. The hypoxic signature of cancer cells poses many problems to the treatment of cancer. Hypoxic tumors are more resistant to chemotherapy, radiation, and even surgery. Additionally, hypoxia induces a number of genes responsible for increased invasion, aggressiveness, and metastases. However, the expression of HIF target genes is not universal and does not always overlap. To compare PDHK1 and CAIX expression, we first employed a Genevestigator analysis of selected genes across public microarray datasets [22] (Figure 1). Among selected genes were four HIF-1 targets: CA9 (carbonic anhydrase IX gene), $P D K 1$ (PDHK1 gene; PDK is an alternative abbreviation for PDHK), PDK3 (PDHK3 gene), and CA12 (transmembrane carbonic anhydrase XII gene). The selection also included related genes, such as CA5A (mitochondrial carbonic anhydrase VA gene), $P D K 2$ and $P D K 4$ (PDHK2 and 4 genes), $P D P 1$ and PDP2 (pyruvate dehydrogenase phosphatase 1 and 2 that activate PDH by dephosphorylation), and CA2 (cytoplasmic carbonic anhydrase II gene). The first evident detail from the comparison is a very similar $C A 9$ and $P D K 1$ gene expression pattern. Both are downregulated or upregulated by the same chemicals, in a number of neoplasms, and in siRNA studies (Figure 1). Interestingly, two other related HIF-1 target genes, PDK3 and CA12, do not share this expression pattern. They are co-expressed with $P D K 1$ and CA9 only in samples where HIF-1 levels are manipulated directly, either by deferoxamine (iron chelator that stabilizes
HIF- $\alpha$ by inhibition of prolyl-hydroxylases) or by silencing of HIF-1a (Figure 1).

Nevertheless, protein expression of PDHK1 and CAIX does not match in healthy tissues according to the Human Integrated Protein Expression Database (HIPED, residing in GeneCards ${ }^{\circ}$, www.genecards.org) created from publiclyavailable mass spectrometry-based proteomics sources. Based on the data, CAIX expression is restricted to the gastrointestinal tract (such as the stomach, cardia, and colon) and secretory tissues (gallbladder, Langerhans islets), while PDHK1 expression is much wider, covering blood cells and immune tissue (B-lymphocytes, CD4 and CD8 T cells, platelets, lymph node, tonsil), frontal cortex, retina, heart, bone, gastrointestinal tissues (esophagus, cardia, stomach, colon,

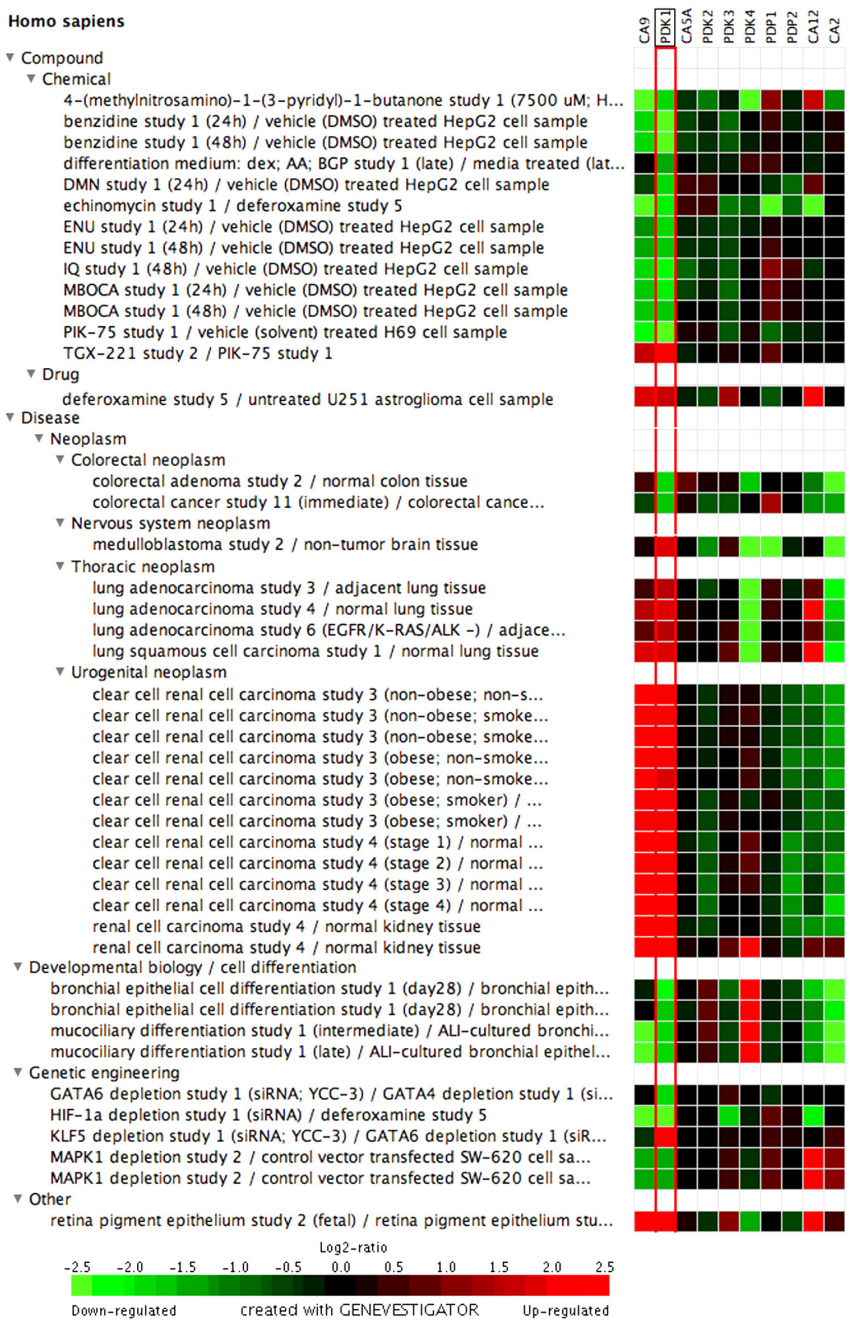

Figure 1. PDHK1 and CAIX are coexpressed in samples from diverse microarray datasets. Expression pattern heat map of CA9 (CAIX gene), PDK1 (PDHK1 gene), CA5A (CAVA gene), PDK2-4 (PDHK2-4 genes), PDP1-2 (PDP1-2 genes), CA12 (CAXII gene), and CA2 (CAII gene) created with Genevestigator from public microarray datasets. Red color signifies upregulation, while green indicates downregulation of expression. 
liver), adipocytes, testis, secretory tissue (salivary gland, adrenal gland, breast, pancreas, placenta, Langerhans islets), and skin. It thus seems that CAIX is a better tumor marker than PDHK1 due to its limited protein expression in healthy tissues. However, it is unknown whether PDHK1 kinase activity towards serine 232 on $\mathrm{PDH}$, which is preferentially induced in hypoxia, couldn't serve as an equally distinctive indicator of malignancy [10].

Effect of PDHK and extracellular CA inhibition on cell viability. Since both PDHK1 and CAIX are important for tumor growth and often co-expressed in different neoplasms, it would be beneficial to try manipulating them in order to reverse tumor adaptation and negatively influence tumor cell proliferation and survival. Reversing tumor adaptation by making cancer cells more normal-like should make them less aggressive or be potentially toxic as such a change shouldn't be able to sustain their proliferation rates. To test this hypothesis, we first treated RKO colorectal carcinoma cells with 4-homosulfanilamide (HSFA, 4-aminomethylbenzenesulfonamide, CAIX and CAXII inhibitor) and dichloroacetate (DCA, pan-PDHK inhibitor), separately and in combination. DCA is a synthetic analogue of pyruvate and a general PDHK1-4 inhibitor (there is no PDHK1-specific inhibitor). PDHK2 and PDHK4 are the most sensitive to DCA, followed by PDHK1 and then PDHK3 [23]. DCA transiently changes glycolytic metabolism to oxidative by activating $\mathrm{PDH}$ through PDHK inhibition. HSFA is a membraneimpermeable sulfonamide analogue, therefore inhibiting only CAs with extracellular catalytic domains, such as CAIX

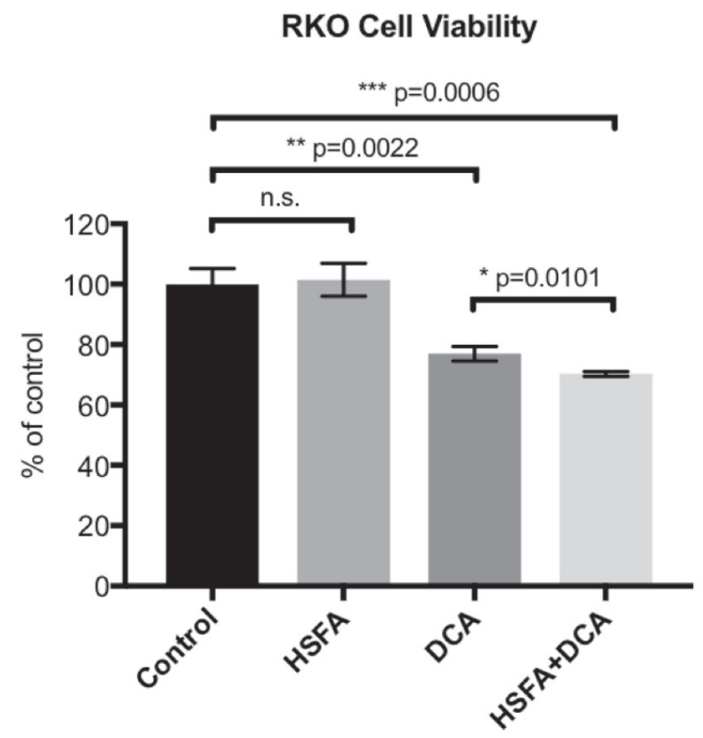

Figure 2. Simultaneous pan-PDHK and transmembrane CA inhibition appears to have a modestly synergistic effect on RKO cell viability. CellTiter-Blue viability assay of RKO cells treated with $100 \mu \mathrm{M}$ homosulfanilamide (HSFA, CAIX and CAXII inhibitor), $10 \mathrm{mM}$ dichloroacetate (DCA, pan-PDHK inhibitor), and their combination for 24 hours (data represented as mean $\pm \mathrm{SD}$; two-tailed Student's t-test). and CAXII [24]. 24-hour treatment with HSFA did not result in reduced viability of RKO cells, while DCA decreased cell viability by $20 \%$ (Figure 2 ). However, when both inhibitors were used together, there was a slightly enhanced effect on viability, with an additional 10\% decrease compared to cells treated with DCA alone (Figure 2).

Expression of PDHK1 and CAIX is mutually influenced. After the encouraging results from pharmacologic inhibition of PDHKs and transmembrane CAs, we decided to prepare PDHK1, CAIX, and double (PDHK1 and CAIX) knockout cells using the CRISPR/Cas9 genome editing technology. We established stable RKO cell lines with knocked-out PDHK1 (PDHK1-/CAIX+), CAIX (PDHK1+/CAIX-), or both (PDHK1-/CAIX-) (Figure 3A). Interestingly, expression of these two proteins was mutually influenced. Absence of PDHK1 led to decreased CAIX expression, while knocking out CAIX similarly reduced PDHK1 levels (Figure 3A). A possible explanation is the absence of HIF-1a transcription factor that induces their expression $[9,14]$ (Figure 3A). Reduced PDHK1 expression in CAIX knockouts however wasn't reflected in decreased phosphorylation of serine 232 on PDH. This PDH-inhibitory site was phosphorylated only in hypoxia (the total PDH level remained the same in all the samples), and only in RKO cells positive for PDHK1. This confirms the previously published results in pancreatic carcinoma cells that PDHK1 is indispensable for PDH serine 232 phosphorylation and is active towards this site specifically under hypoxic conditions, where it significantly reduces $\mathrm{PDH}$ activity and OXPHOS [10].

We were also able to reproduce the effect of PDHK1 downregulation on CAIX expression after silencing of PDHK1 with shRNA in RKO colorectal carcinoma cells (Figure 3B), as well as in the pancreatic carcinoma SU.86.86 cell line (Figure 3C). Interestingly, expression of CAIX was decreased even more than PDHK1 after PDHK1 silencing in RKO cells (Figure 3B) and about the same in SU.86.86 cells (Figure 3C). In both cases, silencing of PDHK1 removed serine 232-PDH phosphorylation almost completely, just like in the case of PDHK1 knockouts, even though the PDHK1 protein levels were still relatively high, especially in RKO cells (Figure 3B). In this case, HIF-1a levels were affected only slightly by PDHK1 silencing, and the decrease was obvious only in low-glucose $(5 \mathrm{mM})$ conditions that reflected in PDHK1 and CAIX expression as well (LG, Figure 3C).

PDHK1 is important for cancer cell survival under nutrient-restricted conditions. We next used our established knockout RKO cancer cell lines in a clonogenic survival assay, where we wanted to see how cells without PDHK1 and/or CAIX would fare under stressful conditions of nutrient deprivation, as are typical for hypoxic tumors because of their poor blood perfusion. We let equal number of cells grow for two weeks in full culture medium $(25 \mathrm{mM}$ glucose, $4 \mathrm{mM}$ glutamine, $1 \mathrm{mM}$ pyruvate) or under nutrient-limited conditions (5 mM glucose, $1 \mathrm{mM}$ gluta- 
A

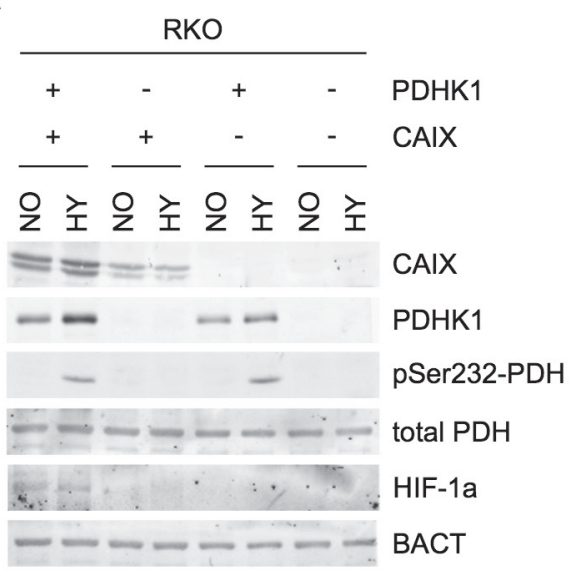

B

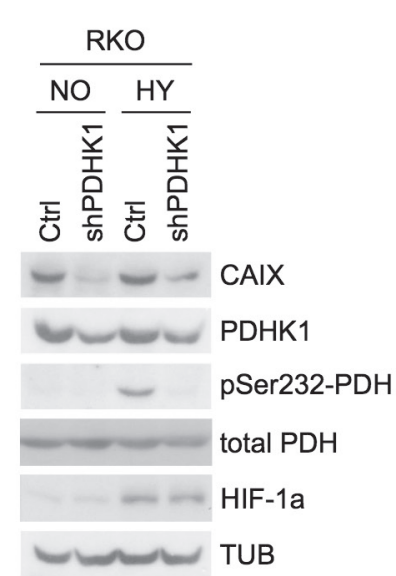

C

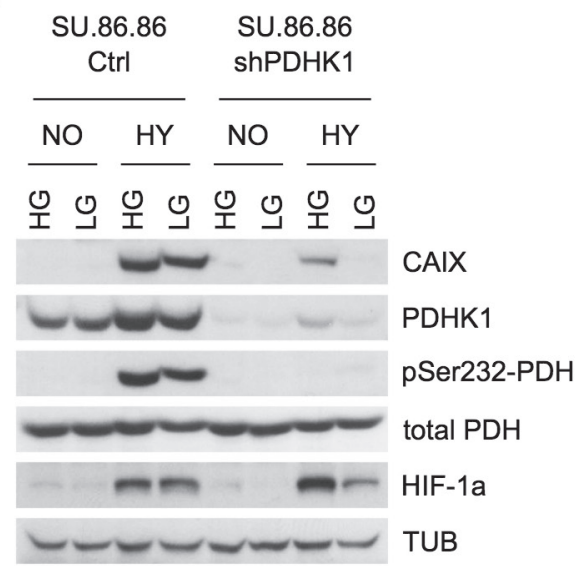

Figure 3. Genetic PDHK1 downregulation decreases CAIX expression. A) Western blots of control (PDHK1+/CAIX+), PDHK1 knockout (PDHK1-/

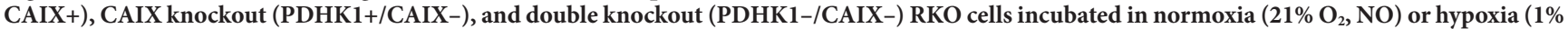
$\mathrm{O}_{2}, \mathrm{HY}$ ) for 16 hours, showing effect on CAIX and PDHK1 expression, HIF-1a levels, and phosphorylation of serine 232 on PDH (pSer232-PDH compared to total PDH, reflecting PDHK1 activity). $\beta$-actin (BACT) served as loading control. Visualized on Li-Cor Odyssey. B) Western blots of control and PDHK1-silenced (shPDHK1) RKO cells incubated in normoxia $\left(21 \% \mathrm{O}_{2}\right.$, NO) or hypoxia $\left(0.5 \% \mathrm{O}_{2}\right.$, $\left.\mathrm{HY}\right)$ for 16 hours, detecting the same antigens as in (A) and confirming decreased CAIX expression after genetic downregulation of PDHK1. $\alpha$-tubulin (TUB) served as loading control. Detected by ECL. C) Western blots of control and PDHK1-silenced (shPDHK1) SU.86.86 cells incubated in normoxia (21\% $\left.\mathrm{O}_{2}, \mathrm{NO}\right)$ or hypoxia $\left(0.5 \% \mathrm{O}_{2}, \mathrm{HY}_{\text {) }}\right.$ in high-glucose ( $25 \mathrm{mM}, \mathrm{HG})$ or low-glucose ( $5 \mathrm{mM}, \mathrm{LG})$ medium for 16 hours, followed by the same detection as in (B).

mine, with or without $1 \mathrm{mM}$ pyruvate), and at the end of the experiment stained surviving colonies with crystal violet for visualization. As can be seen in Figure 4, RKO cells without PDHK1 had much poorer clonogenic survival, independent of CAIX. That points to the importance of PDHK1 in glucose cancer metabolism and its potential value in cancer treatment. The role of CAIX is neutralizing intracellular $\mathrm{pH}$ in an acidic tumor microenvironment, therefore it is unlikely that CAIX would have an effect on clonogenic survival in the conditions of our assay, because the cells had to be grown in normoxia and were very sparse. Therefore, even if they were highly glycolytic, it wouldn't be in their capacity to acidify the ample medium volume. However, it seems that in low glucose, low glutamine conditions (with or without exogenous pyruvate) there was a slight increase in the number of surviving colonies in PDHK1-positive RKO cells with concurrent CAIX expression (PDHK1+/ CAIX+) compared to CAIX knockouts (PDHK1+/CAIX-) (Figure 4). Another interesting point is that without the addition of exogenous pyruvate, more PDHK1-positive RKO cell colonies survived in media with reduced glucose and glutamine concentrations.

PDHK1 and CAIX knockout RKO cells grow slower as xenografted tumors. In the following step, we wanted to see what effect PDHK1 and/or CAIX genetic manipulation would have in vivo, specifically how it would impact xenografted tumor growth in nude mice. We therefore injected subcutaneously the same number of RKO knockout cells into the flanks of nude mice and followed their growth for approximately three weeks (Figure 5A). The control RKO xenografts, positive for both PDHK1 and CAIX, grew signifi- cantly faster than any of the knockouts. It therefore seems questionable if targeting both PDHK1 and CAIX in vivo is really that much more beneficial than just targeting CAIX or PDHK1 individually. However, when we pooled several independent experiments together that differed in the age of experimental animals (6-16 weeks) and in the number of injected cells (2-4 million/tumor), the differences in tumor size weren't as obvious due to the variation within each group, but the double-knockout RKO xenografts (PDHK1-/ CAIX-) still remained the smallest after three weeks of proliferation, followed by CAIX knockouts (PDHK1+/CAIX-) (Figure 5B). Three random tumors from each cell type were also harvested and subjected to Western blot analysis for the detection of CAIX and PDHK1 to confirm their expression (Figure 5C). In the case of PDHK1 knockouts, there is no PDHK1 expression, and we also see concurrently decreased expression of CAIX, just like in 2D cell culture in vitro (Figure $3 \mathrm{~A}$ ). The single bands in CAIX-negative tumor samples represent immunoglobulin from mouse blood and not CAIX (double-band in CAIX-positive tumor samples). Anti-CAIX M75 monoclonal antibody produced in our laboratory is of mouse origin, therefore the secondary anti-mouse antibody used for its detection, also detects the heavy chains of mouse immunoglobulin in the size of CAIX (50-54kDa) (Figure 5C).

Comparison of CAIX and PDHK1 expression in cells originating from primary colorectal tumor and lymphnode metastasis. The SW480 cell line derived from a primary adenocarcinoma of the colon, and the SW620 cell line established from a lymph node metastasis taken from the same patient one year later, served as our model for 


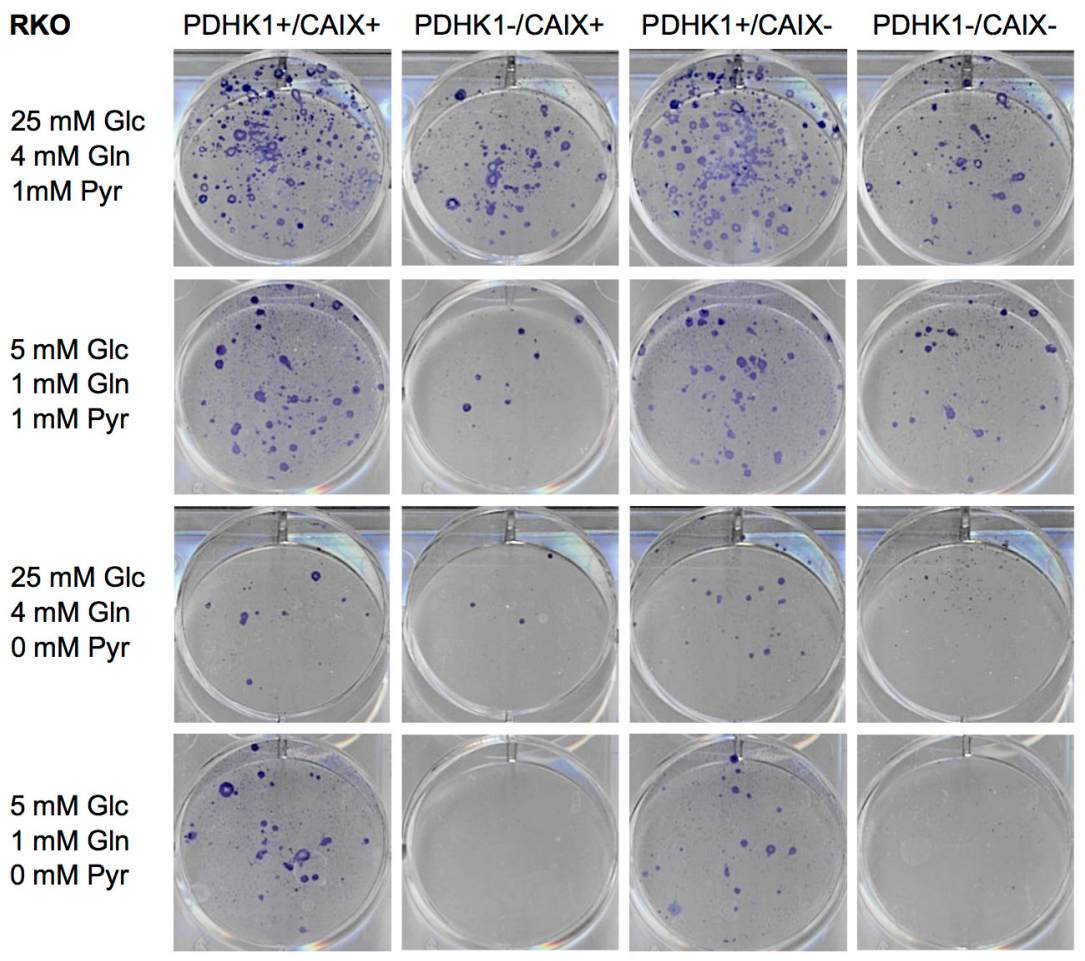

Figure 4. PDHK1 is essential for long-term growth of tumor cells under nutrient-restricted conditions. Photograph of RKO control and PDHK1/CAIX knockout cell colonies stained with crystal violet following growth in complete medium ( $25 \mathrm{mM}$ glucose, $4 \mathrm{mM}$ glutamine) with or without $1 \mathrm{mM}$ pyruvate, or low-glucose $(5 \mathrm{mM})$, low-glutamine $(1 \mathrm{mM})$ medium with or without $1 \mathrm{mM}$ pyruvate for two weeks, showing decreased clonogenic survival of PDHK1-negative cells.

comparison of CAIX and PDHK1 expression in primary tumor vs metastatic cells. We incubated both of these cell lines in normoxia and hypoxia $\left(1 \% \mathrm{O}_{2}\right)$ for 24 hours and after harvesting performed Western blot analysis. Figure 6 shows that the expression of CAIX (normoxia and hypoxia), as well as PDHK1 (hypoxia), is increased in the metastatic SW620 cell line, which suggests that their presence is important for metastatic cells. However, when it comes to PDHK1 activity as estimated by serine 232-PDH phosphorylation, the signal was stronger in SW480 cells from the primary tumor despite having less PDHK1 (Figure 6), which should result in a more pronounced $\mathrm{PDH}$ inhibition, decreased mitochondrial function, and increased glycolysis. Interestingly, the culture medium was also more acidic in the case of hypoxic SW480 than in hypoxic SW620 cells (data not shown), which would also support this assumption, because it is a typical accompanying feature of glycolytic cells producing lactate or of cells with active CAIX [25]. Nevertheless, in both cases, serine 232 of $\mathrm{PDH}$ was phosphorylated even in normoxia, which means that PDHK1 is active in both of these cell types even under those conditions, and these cell lines are therefore more glycolytic than oxidative in general. Expression of another HIF-1 target, LDHA, wasn't affected (Figure 6) but it might have been more active in hypoxic SW480 primary tumor cells due to the acidic culture medium (not shown).

\section{Discussion}

Recent findings in the field of tumor metabolism have brought the Warburg effect [26] back into the scientific spotlight. The unique cancer metabolism exposed several novel pathways that may be targeted thanks to being preferentially used in tumor cells compared to cells from healthy tissue [27]. Cancer cells respond to the distinctive demands within the tumor by systematic changes in their metabolism, and consequently become more viable and resilient. It was this concept that prompted the idea of targeting PDHK1 and CAIX. If these two enzymes were essential for aggressive hypoxic tumors of diverse genetic background, their inhibition may significantly weaken them and make them more vulnerable. In this combination scheme, we aimed to bi-directionally target glycolytic tumor cells and additionally affect cells that may express just one of these two proteins due to tumor heterogeneity. Although the expression of hypoxic target genes usually correlates with HIF-1a in most tumor areas, exceptions have been observed [28]. CAIX for instance is much more stable than HIF-1a [29], which means that cells that had been hypoxic and then reoxygenated may still express CAIX but not HIF-1a. Or cells that have just become hypoxic may be positive for HIF-1a but not yet for CAIX. PDHK1 protein is also very stable after reoxygenation and 
A

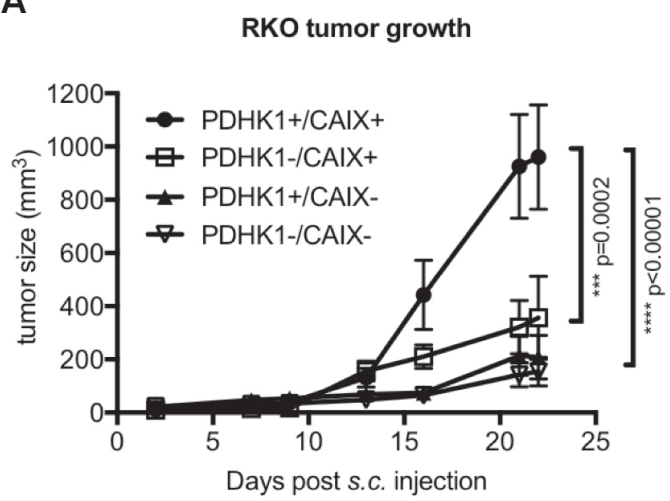

C

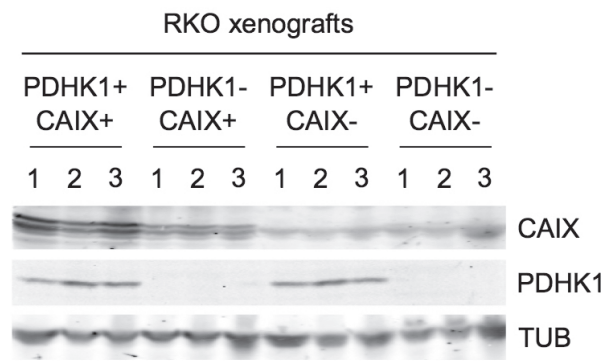

RKO tumor growth Day 21

(3 separate experiments)

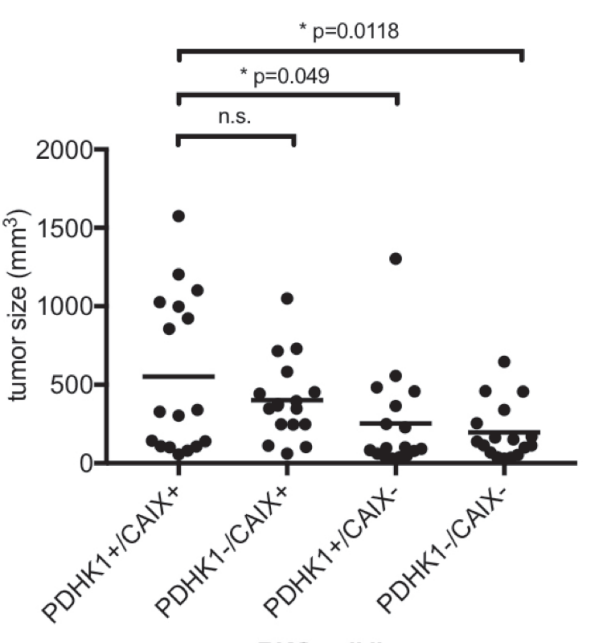

RKO cell lines

Figure 5. Genetic PDHK1 and/or CAIX downregulation significantly slows down growth of xenografted tumors. A) Tumor growth curves of RKO control (PDHK1+/CAIX+), PDHK1 knockout (PDHK1-/CAIX+), CAIX knockout (PDHK1+/CAIX-) or double knockout (PDHK1-/CAIX-) cells grown in 6-week old nude mice (3 x $10^{6}$ cells/tumor). Statistically significant differences exist between control and PDHK1/CAIX knockout tumors as indicated (mean \pm SEM; two-way ANOVA). B) Tumor size of individual RKO tumors from three separate experiments at day 21 (6-16-week old nude mice, $2-4 \times 10^{6}$ cells/tumor). Statistically significant differences exist between control and CAIX-negative tumors (two-tailed Student's t-test). C) Western blots from three random tumors from (B) showing absence of PDHK1 in PDHK1-negative cells and of CAIX in CAIX-negative cells, plus decreased CAIX expression in PDHK1 knockout cells. Single bands in CAIX-positive cells in the size of CAIX represent immunoglobulin heavy chain contamination from mouse blood. a-tubulin (TUB) served as loading control.

is regulated more acutely at the level of activity, which can be visualized by phospho-serine 232-PDH signal that disappears within few hours of reoxygenation [10].

DCA as a general PDHK inhibitor activates PDH and mitochondrial function at the expense of glycolysis. It has been used for several decades to treat children with inborn errors of mitochondrial metabolism and associated lactic acidosis [30]. Carbonic anhydrases are inhibited by sulfonamides used in the treatment of diverse diseases including edema, glaucoma, epilepsy, or infections [31]. Membraneimpermeable derivatives (like HSFA) are thought to inhibit CAs with an extracellular catalytic site, such as the tumorassociated CAIX and CAXII [24]. However, we didn't observe any decrease in viability of RKO cells after incubation with HSFA (Figure 2), although a 24-hour incubation might not be sufficient. DCA as a pan-PDHK inhibitor decreased viability after 24 hours by about 20\% (Figure 2), nevertheless, we used a supra-clinical concentration $(10 \mathrm{mM})$ that we wouldn't be able to achieve in vivo. Yet, there is an interesting paradox for this molecule; although it does not significantly affect cell viability in vitro, there are several preclinical

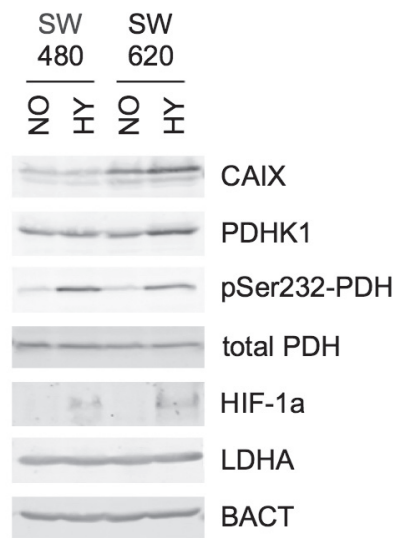

Figure 6. PDHK1 and CAIX appear to be important for primary tumor cells as well as for metastatic cells. Western blots of SW480 colorectal primary tumor cells and matched SW620 metastatic cells incubated in normoxia $\left(21 \% \mathrm{O}_{2}, \mathrm{NO}\right)$ or hypoxia $\left(1 \% \mathrm{O}_{2}, \mathrm{HY}\right)$ for 16 hours, showing increased CAIX and PDHK1 expression in SW620 cells, and increased PDHK1 activity in SW480 cells detected as phosphorylated serine 232 on PDH (pSer232-PDH compared to total PDH). PDHK1 activity towards serine 232 is present also under normoxic conditions in both cell types. LDHA expression remained constant. $\beta$-actin (BACT) served as loading control. Visualized on Li-Cor Odyssey. 
and clinical studies showing its significant effect on tumor growth (summarized in [32]). The reason for this discrepancy probably lies in the different conditions for cells in tissue culture and in tumors themselves. In vitro conditions offer an excess of nutrients from culture media and the available oxygen diffuses to the cells in culture much more easily than in the tumor mass, even under hypoxic conditions [32]. CAIX inhibition as an anti-cancer strategy has also been shown to be significant in a number of preclinical studies on different model tumor types [33-35], and it is possible that HSFA might be more efficient in vivo than in vitro, too. Nonetheless, this experiment at least indicated that by combining PDHK and extracellular CA inhibition, we were able to achieve an additional $10 \%$ decrease in cell viability compared to treatment with DCA alone (Figure 2), which encouraged us to analyze the impact of genetic downregulation of these two proteins, because of the absence of specific and potent inhibitors.

Interestingly, we found that PDHK1 and CAIX mutually influenced each other, and PDHK1 knockout RKO cells had less CAIX, while CAIX knockouts less PDHK1 (Figure 3A). Moreover, we were able to confirm the effect of genetic PDHK1 downregulation on CAIX expression also in an shRNA silencing system (Figures 3B, 3C). It seems that this mutual decrease in expression might be caused by reduced stabilization of HIF- $1 a$ that controls their expression $[9,14]$. The mechanism of HIF- $1 \alpha$ regulation also includes stabilization by glycolytic endproducts, pyruvate and lactate [36], which should both be decreased in PDHK1 knockouts. PDHK1 downregulation activates PDH even in hypoxia (as confirmed by loss of signal for phospho-serine232-PDH, Figure 3), which supports the conversion of pyruvate to acetyl-CoA and on the other hand decreases the conversion of pyruvate to lactate. It has also been analogously reported that DCA (PDHK inhibitor) reduces HIF-1 transactivating activity [37]. In the case of CAIX, it has been shown that its silencing and inhibition improve tumor oxygenation and thus increase radiation sensitivity in vitro and in vivo [34]. Improved oxygenation should lead to HIF-1 1 hydroxylation by prolylhydroxylases and its degradation by the proteasome, which could explain the absence of HIF-1 $\alpha$ in CAIX-negative RKO cells (Figure 3A). We also confirmed that PDHK1 was necessary for serine 232-PDH phosphorylation in the colorectal RKO cell line just like in the previously published pancreatic carcinoma cells, and that PDHK1 activity towards this site was induced by hypoxia (Figure 3A) [10]. Apart from decreased oxygen concentrations, hypoxic tumor areas also display reduced levels of glucose or other nutrients because of the insufficient blood perfusion. When these knockout cells were incubated in culture media with reduced glucose and glutamine concentrations, cells without PDHK1 had a much worse clonogenic survival (independent of CAIX) (Figure 4). Since PDHK1 plays an important role in glucose metabolism, this suggests that RKO cells are dependent on glucose and glycolysis. Glutamine is the second most impor- tant carbon source after glucose for ATP generation and anabolic processes. It also enters the TCA cycle like glucose (at the $a$-ketoglutarate step). However, in a "reversed" TCA cycle, $a$-ketoglutarate can be reductively carboxylated to isocitrate and citrate by isocitrate dehydrogenase 1 (preferred in hypoxia) and contribute to lipid synthesis necessary for tumor cell proliferation [38]. Pyruvate is also commonly added to culture media, even though it is an intermediate in glucose metabolism. Exogenous pyruvate was important even in our experimental setting because the cells had considerably lower clonogenic survival without it (and PDHK1 knockouts had just a few or no surviving colonies), despite high glucose and high glutamine concentrations (Figure 4). It is possible that RKO cells preferentially convert glucose-derived pyruvate to lactate and use glutamine in anabolic processes, making exogenous pyruvate essential for basal functioning of the TCA cycle and OXPHOS. This hypothesis could be supported by the unexpected result that PDHK1-positive RKO cells survived better without exogenous pyruvate in low glucose, low glutamine medium (Figure 4), because high glucose concentrations further stimulate glycolysis at the expense of glucose oxidation in the TCA cycle and OXPHOS, called the Crabtree effect [39]. It is then possible that cancer cells in low glucose medium without exogenous pyruvate are still able to perform basal OXPHOS, while cells with maximally stimulated glycolysis without exogenous pyruvate are not. CAIX contribution to clonogenic survival is not evident in this experiment, likely because CAIX might not even be active in the CAIX-positive cells under normoxic conditions of the experiment. However, when we generated s.c. tumors in nude mice, all of the knockouts grew significantly slower than control RKO cells (Figure $5 \mathrm{~A})$. Many studies describe significant effect of CAIX on the growth of model tumors or formation of metastases [35, $40-42]$. The same is true for PDHK1 [10, 18, 37, 43, 44]. It is therefore questionable if there is any added value in targeting both of these proteins simultaneously when targeting just one significantly slows down model tumor growth. Yet, when we pooled the results of three independent experiments that differed in the age of experimental animals and in the number of injected cells per tumor, we saw that the variation within each cell type was much bigger but the double knockout tumors still remained the smallest, followed closely by CAIX knockouts (Figure 5B). HIF-1 $\alpha$ being upstream of both PDHK1 and CAIX may seem like another possible targeting strategy but evaluation of tumor growth of HIF-1asilenced cells has shown only a modest effect, because HIF-1 controls the expression of many genes, some of which may have opposite impact on tumor growth than PDHK1 and CAIX $[10,45]$.

Additionally, it seems that PDHK1 and CAIX may be important for primary tumors in respect to their enzymatic activity (acute effect), as well as for metastases because of their high expression (chronic effect) (Figure 6). Increased CAIX expression in the SW620 metastatic cancer cells may 
reflect the role of CAIX in cancer cell deadhesion necessary for metastasis, which is not only related to its ability to affect $\mathrm{pH}$ but CAIX is also able to reduce E-cadherinmediated adhesion by binding to $\beta$-catenin [46]. Increased PDHK1 activity in the primary tumor SW480 cells suggests they are able to acutely respond to the fluctuating conditions within the tumor mass. All in all, our results indicate that targeting PDHK1 and CAIX could be beneficial in the treatment of hypoxic tumors that tend to be resistant to standard therapy. It would be important however to identify patients with increased tumor CAIX expression and PDHK1 activity (through phospho-serine 232-PDH), who could benefit from the targeted strategy. It is equally important that new specific and potent inhibitors of CAIX and PDHK1 are designed that could be used in such metabolically-targeted treatment, either by themselves or in combination with standard cytotoxic agents, the effective dosage of which could be then potentially decreased in order to prevent their serious adverse side effects that often lead to septic shock and death.

Acknowledgements: This work was co-financed by the EU and SAS (SASPRO Programme grant 0035/01/02) and the Slovak Scientific Grant Agency VEGA (2/0155/15; 2/0122/16).

\section{References}

[1] BROWN JM, WILSON WR. Exploiting tumor hypoxia in cancer treatment. Nat Rev Cancer 2004; 4: 437-447. https:// doi.org/10.1038/nrc1367

[2] HUANG LE, GU J, SCHAU M, BUNN HF. Regulation of hypoxia-inducible factor 1alpha is mediated by an O2-dependent degradation domain via the ubiquitin-proteasome pathway. Proc Natl Acad Sci U S A 1998; 95: 7987-7992.

[3] SCHOFIELD CJ, RATCLIFFE PJ. Oxygen sensing by HIF hydroxylases. Nat Rev Mol Cell Biol 2004; 5: 343-354. https:// doi.org/10.1038/nrm1366

[4] LUNT SY, VANDER HEIDEN MG. Aerobic glycolysis: meeting the metabolic requirements of cell proliferation. Annu Rev Cell Dev Biol 2011; 27: 441-464. https://doi. org/10.1146/annurev-cellbio-092910-154237

[5] DENKO NC. Hypoxia, HIF1 and glucose metabolism in the solid tumor. Nat Rev Cancer 2008; 8: 705-713. https://doi. org/10.1038/nrc2468

[6] VANDER HEIDEN MG, CANTLEY LC, THOMPSON CB. Understanding the Warburg effect: the metabolic requirements of cell proliferation. Science 2009; 324: 1029-1033. https://doi.org/10.1126/science.1160809

[7] KIM JW, TCHERNYSHYOV I, SEMENZA GL, DANG CV. HIF-1-mediated expression of pyruvate dehydrogenase kinase: a metabolic switch required for cellular adaptation to hypoxia. Cell Metab 2006; 3: 177-185. https://doi. org/10.1016/j.cmet.2006.02.002

[8] LU CW, LIN SC, CHEN KF, LAI YY, TSAI SJ. Induction of pyruvate dehydrogenase kinase- 3 by hypoxia-inducible factor-1 promotes metabolic switch and drug resistance. J Biol Chem 2008; 283: 28106-28114. https://doi.org/10.1074/jbc. M803508200
[9] PAPANDREOU I, CAIRNS RA, FONTANA L, LIM AL, DENKO NC. HIF-1 mediates adaptation to hypoxia by actively downregulating mitochondrial oxygen consumption. Cell Metab 2006; 3: 187-197. https://doi.org/10.1016/j. cmet.2006.01.012

[10] GOLIAS T, PAPANDREOU I, SUN R, KUMAR B, BROWN $\mathrm{NV}$ et al. Hypoxic repression of pyruvate dehydrogenase activity is necessary for metabolic reprogramming and growth of model tumors. Sci Rep 2016; 6: 31146. https://doi. org/10.1038/srep31146

[11] FIRTH JD, EBERT BL, PUGH CW, RATCLIFFE PJ. Oxygenregulated control elements in the phosphoglycerate kinase 1 and lactate dehydrogenase A genes: similarities with the erythropoietin 3' enhancer. Proc Natl Acad Sci U S A 1994; 91: 6496-6500. https://doi.org/10.1073/pnas.91.14.6496

[12] TANNOCK IF, ROTIN D. Acid pH in tumors and its potential for therapeutic exploitation. Cancer Res 1989; 49: 4373-4384.

[13] SHIMODA LA, FALLON M, PISARCIK S, WANG J, SEMENZA GL. HIF-1 regulates hypoxic induction of NHE1 expression and alkalinization of intracellular $\mathrm{pH}$ in pulmonary arterial myocytes. Am J Physiol Lung Cell Mol Physiol 2006; 291: L941-949. https://doi.org/10.1152/ajplung.00528.2005

[14] WYKOFFCC,BEASLEYNJ,WATSONPH,TURNERKJ,PASTOREK J et al. Hypoxia-inducible expression of tumor-associated carbonic anhydrases. Cancer Res 2000; 60: 7075-7083.

[15] SVASTOVA E, WITARSKI W, CSADEROVA L, KOSIK I, SKVARKOVA L et al. Carbonic anhydrase IX interacts with bicarbonate transporters in lamellipodia and increases cell migration via its catalytic domain. J Biol Chem 2012; 287: 3392-3402. https://doi.org/10.1074/jbc.M111.286062

[16] POTTER CP, HARRIS AL. Diagnostic, prognostic and therapeutic implications of carbonic anhydrases in cancer. Br J Cancer 2003; 89: 2-7. https://doi.org/10.1038/sj.bjc.6600936

[17] WIGFIELD SM, WINTER SC, GIATROMANOLAKI A, TAYLOR J, KOUKOURAKIS ML et al. PDK-1 regulates lactate production in hypoxia and is associated with poor prognosis in head and neck squamous cancer. Br J Cancer 2008; 98: 1975-1984. https://doi.org/10.1038/sj.bjc.6604356

[18] KOUKOURAKIS MI, GIATROMANOLAKI A, SIVRIDIS E, GATTER KC, HARRIS AL et al. Pyruvate dehydrogenase and pyruvate dehydrogenase kinase expression in non small cell lung cancer and tumor-associated stroma. Neoplasia 2005; 7: 1-6.

[19] CHAE YC, VAIRA V, CAINO MC, TANG HY, SEO JH et al. Mitochondrial Akt Regulation of Hypoxic Tumor Reprogramming. Cancer Cell 2016; 30: 257-272. https://doi. org/10.1016/j.ccell.2016.07.004

[20] DITTE P, DEQUIEDT F, SVASTOVA E, HULIKOVA A, OHRADANOVA-REPIC A et al. Phosphorylation of carbonic anhydrase IX controls its ability to mediate extracellular acidification in hypoxic tumors. Cancer Res 2011; 71: 75587567. https://doi.org/10.1158/0008-5472.CAN-11-2520

[21] LI X, JIANG Y, MEISENHELDER J, YANG W, HAWKE $\mathrm{DH}$ et al. Mitochondria-Translocated PGK1 Functions as a Protein Kinase to Coordinate Glycolysis and the TCA Cycle in Tumorigenesis. Mol Cell 2016; 61: 705-719. https://doi. org/10.1016/j.molcel.2016.02.009 
[22] HRUZ T, LAULE O, SZABO G, WESSENDORP F, BLEULER $S$ et al. Genevestigator v3: a reference expression database for the meta-analysis of transcriptomes. Adv Bioinformatics 2008; 2008: 420747. https://doi.org/10.1155/2008/420747

[23] STACPOOLE PW. The pharmacology of dichloroacetate. Metabolism 1989; 38: 1124-1144. https://doi.org/10.1016/00260495(89)90051-6

[24] HULIKOVA A, SWIETACH P. Rapid CO2 permeation across biological membranes: implications for $\mathrm{CO} 2$ venting from tissue. FASEB J 2014; 28: 2762-2774. https://doi. org/10.1096/fj.13-241752

[25] SVASTOVA E, HULIKOVA A, RAFAJOVA M, ZAT'OVICOVA M, GIBADULINOVA A et al. Hypoxia activates the capacity of tumor-associated carbonic anhydrase IX to acidify extracellular pH. FEBS Lett 2004; 577: 439-445. https://doi.org/10.1016/j.febslet.2004.10.043

[26] WARBURG O, WIND F, NEGELEIN E. The Metabolism of Tumors in the Body. J Gen Physiol 1927; 8: 519-530.

[27] PANISOVA E, KERY M, KOPACEK J, PASTOREKOVA S, SVASTOVA E. Enhanced metabolism as a common feature of cancer plasticity. Neoplasma 2016; 63: 836-845. https:// doi.org/10.4149/neo_2016_602

[28] MAYER A, HOCKEL M, VAUPEL P. Carbonic anhydrase IX expression and tumor oxygenation status do not correlate at the microregional level in locally advanced cancers of the uterine cervix. Clin Cancer Res 2005; 11: 7220-7225. https:// doi.org/10.1158/1078-0432.CCR-05-0869

[29] RAFAJOVA M, ZATOVICOVA M, KETTMANN R, PASTOREK J, PASTOREKOVA S. Induction by hypoxia combined with low glucose or low bicarbonate and high posttranslational stability upon reoxygenation contribute to carbonic anhydrase IX expression in cancer cells. Int J Oncol 2004; 24: 995-1004.

[30] STACPOOLE PW, KURTZ TL, HAN Z, LANGAEE T. Role of dichloroacetate in the treatment of genetic mitochondrial diseases. Adv Drug Deliv Rev 2008; 60: 1478-1487. https:// doi.org/10.1016/j.addr.2008.02.014

[31] SUPURAN CT. Carbonic anhydrases: novel therapeutic applications for inhibitors and activators. Nat Rev Drug Discov 2008; 7: 168-181. https://doi.org/10.1038/nrd2467

[32] PAPANDREOU I, GOLIASOVA T, DENKO NC. Anticancer drugs that target metabolism: Is dichloroacetate the new paradigm? Int J Cancer 2011; 128: 1001-1008. https://doi. org/10.1002/ijc. 25728

[33] AHLSKOG JK, DUMELIN CE, TRUSSEL S, MARLIND J, NERI D. In vivo targeting of tumor-associated carbonic anhydrases using acetazolamide derivatives. Bioorg Med Chem Lett 2009; 19: 4851-4856. https://doi.org/10.1016/j. bmcl.2009.06.022

[34] DUBOIS L, PEETERS S, LIEUWES NG, GEUSENS N, THIRY A et al. Specific inhibition of carbonic anhydrase IX activity enhances the in vivo therapeutic effect of tumor irradiation. Radiother Oncol 2011; 99: 424-431. https://doi. org/10.1016/j.radonc.2011.05.045
[35] LOU Y, MCDONALD PC, OLOUMI A, CHIA S, OSTLUND $\mathrm{C}$ et al. Targeting tumor hypoxia: suppression of breast tumor growth and metastasis by novel carbonic anhydrase IX inhibitors. Cancer Res 2011; 71: 3364-3376. https://doi. org/10.1158/0008-5472.CAN-10-4261

[36] LU H, FORBES RA, VERMA A. Hypoxia-inducible factor 1 activation by aerobic glycolysis implicates the Warburg effect in carcinogenesis. J Biol Chem 2002; 277: 23111-23115. https://doi.org/10.1074/jbc.M202487200

[37] KINNAIRD A, DROMPARIS P, SALEME B, GURTU V, WATSON K et al. Metabolic Modulation of Clear-cell Renal Cell Carcinoma with Dichloroacetate, an Inhibitor of Pyruvate Dehydrogenase Kinase. Eur Urol 2016; 69: 734-744. https://doi.org/10.1016/j.eururo.2015.09.014

[38] METALLO CM, GAMEIRO PA, BELL EL, MATTAINI KR, YANG J et al. Reductive glutamine metabolism by IDH1 mediates lipogenesis under hypoxia. Nature 2011; 481: 380-384. https://doi.org/10.1038/nature10602

[39] BLOCH-FRANKENTHAL L, RAM D. The relationship between the Crabtree effect and the oxidative metabolism of glucose and carbohydrate intermediates in ascites tumor cells. Cancer Res 1959; 19: 835-842.

[40] MCINTYRE A, PATIAR S, WIGFIELD S, LI JL, LEDAKI I et al. Carbonic anhydrase IX promotes tumor growth and necrosis in vivo and inhibition enhances anti-VEGF therapy. Clin Cancer Res 2012; 18: 3100-3111. https://doi. org/10.1158/1078-0432.CCR-11-1877

[41] ROBERTSON N, POTTER C, HARRIS AL. Role of carbonic anhydrase IX in human tumor cell growth, survival, and invasion. Cancer Res 2004; 64: 6160-6165. https://doi. org/10.1158/0008-5472.CAN-03-2224

[42] WARD C, MEEHAN J, MULLEN P, SUPURAN C, DIXON JM et al. Evaluation of carbonic anhydrase IX as a therapeutic target for inhibition of breast cancer invasion and metastasis using a series of in vitro breast cancer models. Oncotarget 2015; 6: 24856-24870. https://doi.org/10.18632/ oncotarget. 4498

[43] MCFATE T, MOHYELDIN A, LU H, THAKAR J, HENRIQUES J et al. Pyruvate dehydrogenase complex activity controls metabolic and malignant phenotype in cancer cells. J Biol Chem 2008; 283: 22700-22708. https://doi.org/10.1074/ jbc.M801765200

[44] PENG F, WANG JH, FAN WJ, MENG YT, LI MM et al. Glycolysis gatekeeper PDK1 reprograms breast cancer stem cells under hypoxia. Oncogene 2018; 37: 1119. https://doi. org/10.1038/onc.2017.407

[45] LI L, LIN X, STAVER M, SHOEMAKER A, SEMIZAROV $\mathrm{D}$ et al. Evaluating hypoxia-inducible factor-1alpha as a cancer therapeutic target via inducible RNA interference in vivo. Cancer Res 2005; 65: 7249-7258. https://doi. org/10.1158/0008-5472.CAN-04-4426

[46] SVASTOVA E, ZILKA N, ZAT'OVICOVA M, GIBADULINOVA A, CIAMPOR F et al. Carbonic anhydrase IX reduces E-cadherin-mediated adhesion of MDCK cells via interaction with beta-catenin. Exp Cell Res 2003; 290: 332-345. https://doi.org/10.1016/S0014-4827(03)00351-3 